\title{
Relationships and Responsibilities of Software Experimentation
}

\author{
Giovanni Cantone
}

The Empirical software engineering (ESE) research community is twenty years old but still growing mainly on the internal side rather than in terms of external relationships. Let us reason on the most important of those ESE relationships and consequent responsibilities. We sketch on a static view of the problem domain entities, and their relationships, in case stereotyped relationships.

ESE Research Groups aim to improve Organizations, a term that generalizes on Developers, Producers, and Service-agencies. Organizations enact their own Processes, use Methods, utilize Tools, and employ People. Associations of (types of) Organizations do produce Standards, and (Production I Development I Service) Guidelines, including Good Production I Development Practices. The main goal is to filter products, hence organizations, which are allowed for a certain market.

Some Products, Methods, Tools are for use or consume by End-customers or for use in the processes enacted by Agencies, which provide services to organizations and citizens, who generally are not aware of the details concerning the short and long term impacts of that technology on themselves and the environment. Hence, the need and role of Authorities, which enact Ethical Control, and emit Guidelines; these include Verification guidelines and Good Laboratory Practices, (GLP)s. Because the labs of an Organization live a context that applies standards and good practices, they usually also apply (GLP)s. Vice versa, concerning other types of labs, e.g. academic labs, the application of those practices probably vary from context to context.

Software organizations are development organizations. They develop technology for end-customers and all types of organizations. Hence, there are three relevant types of software technology transfer: from software developer to software developer, or producer, or service provider, respectively. Concerning the latter, for instance, software technology has been proposed for the continual learning of physicians and paramedics. ESE should hence investigate all those types of technology transfer.

The ESE research has dependence relationships with Ethical control, (GLP)s, and related Guidelines. In other fields there are many types of experiments, to conduct orderly (in case of success only!) E0 acts on things, "in-vitro"; experimenters are the only humans allowed to come in contact; ESE scientists should simulate their experiment processes before involving volunteers. E1 acts on primary animals; seems not applicable for ESE experiments. E2 acts on formally informed human volunteers performing as subjects. $E_{n>2}$ act on dependent humans: the lower is $n$ the greater is the level of dependency of the involved subjects. Based on the ESE practices, to the best or our knowledge, ESE experiments usually skip the stages E0, and still limit to enact (part of) E2, which is a quite frustrating fact that we should work to remove. 